\title{
Homonacionalismo en Cataluña: una visión desde el activismo LGTBI*
}

\section{Homonationalism in Catalonia: an approach from LGTBI activism}

Recibido: 5 de noviembre de 2014 | Aceptado: 15 de agosto de 2015

\author{
NúRIA SADURNí BALCELLS ** \\ JOAN PUJOL TARRÉS ${ }^{* * *}$ \\ Universitat Autònoma de Barcelona, Cataluña, España
}

doi :10.11144/Javeriana.upsy14-5.hcva

Para citar este artículo: Sadurní, N., \& Pujol, J. (2015). Homonacionalismo en Cataluña: una visión desde el activismo LGTBI. Universitas Psychologica, 14(5), 1809-1820. http://dx.doi.org/10.11144/ Javeriana.upsy14-5.hcva

Artículo de reflexión. Este trabajo ha sido realizado en el marco del programa de Doctorado en Persona y Sociedad en el Mundo Contemporáneo de la Universitat Autònoma de Barcelona.

** Estudiante de doctorado. Grupo de Investigación en Investigación Crítica (FIC). Departamento de Psicología Social. Correo electrónico: nuria. sadurni@e-campus.uab.cat

**** Doctor en Psicología Social. Profesor Titular. Departamento de Psicología Social. Grupo de investigación Fractalidades en Investigación Crítica (FIC). Correo electrónico: Joan.Pujol@uab.cat

\section{RESUMEN}

El homonacionalismo constituye un marco conceptual que permite comprender cómo las políticas LGTB han sido instrumentalizadas en la construcción de una diferenciación geopolítica de carácter colonial. Construye relaciones de equivalencia entre las posiciones de sujeto 'homosexual' y 'nacional' que permite una diferenciación intra-nacional y entre-naciones de sujetos y poblaciones, justificando políticas de afirmación con base en el avance en algunos de los derechos humanos. Este fenómeno ha sido identificado principalmente en las políticas LGTB de EE. UU. y su desarrollo teórico se está extendiendo por el mundo. Ante los recientes desarrollos, tanto en términos de identidad nacional como de prácticas y marcos legales en defensa de los derechos LGTB, Cataluña podría constituir un territorio propicio para la construcción de un excepcionalismo que alimente discursos homonacionalistas. En este trabajo, se explora este tipo de discurso en narrativas construidas con activistas de grupos LGTBI en Barcelona, constatando su presencia y la consolidación de una normatividad que es cuestionada por las activistas.

Palabras clave

homonacionalismo; feminismo poscolonial; colectivos LGTBI; producciones narrativas

\footnotetext{
A B S T R A C T

Homonationalism is a conceptual framework that allows to understand how the struggle for LGTB rights is being assimilated by national exceptionalism in order to constitute a geopolitical colonial differentiation. It builds equivalences between homosexual and national subject positions that allows the differentiation of subject positions and populations within the country and between countries, justifying policies based on the differentiation of human rights development. This phenomenon has been applied to the US and its theoretical development is being spreading throughout the world. In light of recent developments both in terms of national identity and LGTB legal rights, Catalonia could be susceptible of an exceptionalism that could lead to homonationalist discourses. This paper explores the homonationalist discourse in Catalonia using narratives from LGTBI activists. Participants identify a homonationalist discourse and recognise the presence of homonormativity questioned by the activist. Nevertheless, a homonationalist geopolitical hierarchy is not appreciated.

Keywords

homonationalism; postcolonial feminism; LGTBI groups; narrative productions
} 


\section{Posiciones de sujeto y acción política interseccional}

Los movimientos feministas, LGTBI ${ }^{1}$ y étnicos (i. e., movimiento indígena) forman parte de lo que se denomina "nuevos movimientos sociales" que dan importancia no solo al reconocimiento, sino además a la mejora de las condiciones materiales. Al ser movimientos en los que la constitución de sujeto es elemento fundamental, son a menudo considerados, aunque sea parcialmente, "movimientos identitarios". Género, sexualidad y pertenencia étnica conforman fuentes fundamentales de constitución identitaria y diferenciación social (Anthias, 1999), formas de "construcción del yo" que se han fortalecido junto a la consolidación de las sociedades del conocimiento (Castells, 1999). Se trata de movimientos de resistencia que inciden en los aspectos semióticos y simbólicos, por lo que el desarrollo de un concepto más amplio de capital ha permitido su revalorización por parte de perspectivas posmarxistas (Bourdieu, 1986, 1996; Wacquant, 2006). El análisis de la desigual distribución del capital económico y simbólico entre distintos grupos sociales permite sostener una perspectiva interseccional (Crenshaw, 1991; Valentine, 2007) que tiene en cuenta tanto el capital económico como el capital simbólico.

La importancia de la constitución histórica del sujeto en tanto que agente de transformación social deviene un elemento central de captura por parte del poder (Adorno, 1990, 1991; Bourdieu, 2002). Sin embargo, ihasta qué punto un sujeto que es constituido por unas estructuras sociales internalizadas puede escapar de la reproducción de estas mismas estructuras? La transformación social desde una cierta posición de sujeto no puede pensarse fuera de las relaciones sociales que constituye esa posición de sujeto (Durand Ponte \& Cuéllar Vázquez, 1989). Es necesario entonces pensar en un espacio

1 LGTBI: lesbianas, gais, transexuales, bisexuales e intersexuales. En este texto, se usan distintas siglas dependiendo del contexto narrativo y de lo que se incluye en el término. Usamos LGTBI cuando nos referimos al movimiento en general y LGT/LGTB en referencia a luchas, derechos y/o identidades concretas. social heterogéneo, múltiple, con distintas intensidades, temporalidades y espacialidades. Un espacio con múltiples relaciones de equivalencia (Laclau \& Mouffe, 1985) entre las distintas posiciones, dentro y entre los distintos campos, donde se conforma un campo de conflicto en el que anclan nuevos significados y prácticas (Gramsci, 1975; Gunn, 2006). Constituirse como sujeto de resistencia implica entrar en un campo de relaciones de equivalencia y/o conflicto frente a otras posiciones del campo. Para que una cierta posición de sujeto tenga incidencia social es necesario que se involucre interseccionalmente con otras posiciones de resistencia. Al mismo tiempo, los estamentos de poder desarrollan estrategias para capturar estas posiciones de sujeto. Precisamente, el concepto de homonacionalismo (Puar, 2007) habla de la captura de la posición de sujeto LGTBI por parte de estructuras nacionales, dentro de un esquema colonial de reforzamiento de las divisiones geopolíticas en el que la aceptación y tolerancia hacia esa población se convierte en indicador de la madurez democrática y capacidad de soberanía nacional (Andrade, 2014).

\section{Homonacionalismo y reconfiguración nacional de posiciones críticas de sujeto}

Tradicionalmente, los análisis feministas y LGTBI han caracterizado al Estado como inherentemente 'heteronormativo' y a las identidades LGTBI como constituidas fuera del Estado nación (Puar, 2013). Así mismo, el feminismo blanco ha obviado la articulación del racismo en la teoría y en la práctica, centrándose prácticamente de forma exclusiva en el género y la sexualidad (Lugones, 2007). Sin embargo, la progresiva inclusión de los derechos LGTB dentro del aparato legal y normativo de los denominados países "desarrollados" ha permitido el establecimiento de una jerarquía geopolítica que marca el nivel de modernidad y madurez de un país con base en la aceptación de los derechos LGTB (Puar, 2007), creando así fuertes efectos racistas. El "homonacionalismo" constituye una propuesta analítica para comprender y contextualizar históricamente la deseabilidad de incluir los derechos LGTB dentro del marco legal de los Estados nación (Puar, 
2007, 2013). De la misma forma que la apropiación estatal del discurso feminista en relación con el tratamiento de las mujeres por parte del movimiento talibán justificó la intervención militar en Afganistán, la apropiación del discurso LGTBI configuraría un nacionalismo hetero/homo-normativo liberal y tolerante que incluiría la multiculturalidad, el reconocimiento de los derechos LGTB y el respeto a múltiples prácticas sexuales a la vez que constituiría otredades étnico-nacionales justificativas de acciones geopolíticas basadas, precisamente, en la falta de reconocimiento de los derechos de grupos minoritarios (Puar, 2006). La articulación de las identidades lesbianas y gay, en lugar de dirigirse al cuestionamiento de las asunciones heteronormativas, supondría una reproducción de la heteronormatividad (Ammaturo, 2014), donde se accedería a una serie de privilegios excluyentes. El homonacionalismo es, pues, un movimiento biopolítico que recupera ciertos cuerpos homosexuales como parte del cuerpo de la nación (Haritaworn, 2013), y lo hace en oposición a sujetos localizados geopolíticamente construidos en términos de incapacidad de reconocer los derechos de las minorías sexuales (Lamble, 2014). A través de esta lógica, la inclusión de la homosexualidad en el cuerpo de la nación consolida una división geopolítica entre civilización gay-friendly ${ }^{2}$ y barbarie (homófoba), que sitúa a las comunidades pensadas como "Primer Mundo" en terreno civilizado, mientras que otras son ubicadas en el territorio de la homofobia y la barbarie, especialmente las musulmanas (Puar, 2007). Se refuerza de este modo un eje colonial que, como señala la feminista decolonial Ochy Curiel: "Hoy la alteridad [...] sigue siendo 'materia prima' para el colonialismo occidental, [...] que sigue siendo patriarcal, además de racista" (Curiel, 2007, p. 100).

Aunque el fenómeno del homonacionalismo es ejemplificado en el contexto estadounidense, varias autoras han explorado su presencia en diferentes partes del mundo y toman el concepto para pensar sobre lo queer en otros contextos que excedan los de

2 Gay-friendy es un préstamo lingüístico del inglés que se utiliza para referirse a actitudes, establecimientos o instituciones amigables para las personas LGTB. las grandes ciudades en los llamados "países centrales" (Ferreira, 2014). Por ejemplo, en distintos países de Latinoamérica se está valorando la articulación teórica del homonacionalismo con los debates de la teoría feminista y queer latinoamericanos (Seguer, 2014) y se está aplicando para analizar aspectos de ciertas demandas LGTB (Andrade, 2014). Sin embargo, es en Estados Unidos, Canadá y Europa donde de momento encontramos el grueso bibliográfico sobre homonacionalismo.

\section{Homonacionalismo en Cataluña}

Partiendo de este concepto, nos interesaba ver qué posibles articulaciones podría haber entre el desarrollo teórico del homonacionalismo por parte de varias autoras ${ }^{3}$ (aunque principalmente a partir de Jasbir Puar) y la situación en Cataluña, actualmente parte del Estado español.

El conocido eslogan turístico de los años 60, "Spain is different", señala la excepcionalidad española (Townson, 2011) construida en torno a un cierto complejo de inferioridad respecto a Europa (Roig Tapia, 2009, p. 46). El nacionalismo catalán, por otra parte, construye su excepcionalismo y singularidad en contraposición a España y en línea con el resto de Europa (Gil Calvo, 2006). Si bien Cataluña forma parte del Estado español, nos encontramos con un fuerte movimiento independentista basado en la diferenciación respecto a él y en línea con los imaginarios de primer mundo que habitan la Unión Europea, en un contexto donde Cataluña aspira a ser una nación independiente de España -un catalanismo político que define a Cataluña como lugar de acogida y multiculturalidad (Domingo, 2013) a la vez que parte fundamental de la Unión Europea-. La relevancia y presencia de los movimientos LGTBI en Cataluña junto al desarrollo del excepcionalismo catalán asociado al desarrollo de los movimientos nacionalistas hace

3 Para no utilizar el masculino de forma genérica, y para evitar discriminaciones por razones de género, $\mathrm{y}$ a falta de una normativa en lengua castellana para utilizar una forma genérica sin marca de género, en este artículo utilizaré el femenino genérico como si estuviera hablando siempre del sujeto omitido "persona". 
plausible la presencia de formas de homonacionalismo dentro del discurso nacional catalán.

El excepcionalismo supone una autodistinción positiva respecto a un/os otro/s constitutivos, y cuando es nacional, habla de la excelencia de la propia nación y de la distinción y superioridad de su población frente a otras entidades nacionales (Puar, 2007). Como extensión del mismo, el excepcionalismo sexual hace referencia a la distinción social y legal con respecto a los derechos sexuales y a la disidencia sexual en comparación a otros territorios. Esta forma de excepcionalismo funciona como reguladora de las fronteras de lo aceptable en género, raza y clase (Puar, 2007), al designar su forma de aceptación de la sexualidad como la buena, la excepcional. Aunque no hay estudios importantes sobre este tema en Cataluña, el discurso sobre una nación independiente se basa en la inclusión e identificación con Europa, como muestra la campaña "Cataluña, nuevo Estado en Europa".

Al día de hoy, prácticamente no se cuenta con trabajos hechos sobre homonacionalismo en Cataluña, pero en tratándose de una región que se sitúa a sí misma en línea con Europa, podemos pensar a priori que es posible la parte que se identifica con Europa tenga grandes semejanzas con lo que se podría encontrar en Cataluña. En Europa, varias identifican claramente la presencia de discursos y prácticas homonacionalistas, considerándolo un fenómeno generalizado en el continente (El-Tayeb, 2012) y un territorio con unas libertades progresistas que se deben proteger, " $a$ fortress of sexual freedom under seige" (Colpani \& Habed, 2014, p. 13).

Por este motivo, nos interesaba buscar elementos de donde se deduce que hay prácticas y discursos homonacionalistas en Cataluña y cómo esta se puede construir como un lugar de excepcionalidad sexual. Además, quisimos explorar las relaciones entre homonacionalismo e independentismo catalán, así como analizar sus prácticas y las diferentes alteridades que se producen en el discurso, en Cataluña. Partiendo de la premisa de que las activistas LGTBI deberían tener una mayor exposición a los

4 "Una fortaleza de libertad sexual bajo asedio" [traducción propia]. discursos conectados con el concepto de homonacionalismo, este trabajo explora el vínculo entre aquellos -sobre migración en activistas LGTBI-y dicho concepto con el objetivo de tener una primera impresión de la extensión de este discurso en Cataluña.

El método utilizado en esta investigación han sido las producciones narrativas (Balasch \& Montenegro, 2003). Se trata de una técnica basada en la epistemología de los conocimientos situados (Haraway, 1991) que, reconociendo la parcialidad de la mirada y la necesidad de articularse con otras posiciones de sujeto, explora la posición de la participante frente a un fenómeno determinado, a partir de varias conversaciones entre investigadora y participante. En una primera fase, registramos la conversación con las participantes alrededor de diversos temas. Hablamos sobre excepcionalismo sexual, lo queer como regulador, la predominancia blanca, necropolítica queer y la relación entre las luchas LGTB y el nacionalismo heteronormativo. Después de estas conversaciones, textualizamos la conversación y esta se retornó a las participantes, abriendo nuevos interrogantes y observaciones tanto por parte de la participante como de la investigadora. Después de distintos encuentros y retoques del texto, finalmente llegamos a una versión final que es a la que llamamos producción narrativa o simplemente narrativa. La posibilidad de leerla y modificarla, otorga una agencia a las participantes que es especialmente valorada por personas involucradas con el activismo LGTBI (MartínezGuzmán \& Montenegro, 2010). La versión final estuvo validada por cada participante porque en esta metodología "en lugar de representar cómo las participantes comprenden el fenómeno, [se] busca expresar cómo quieren que un particular tema sea visto" (Pujol, Montenegro, \& Balasch, 2003, p. 67). Las narrativas no fueron posteriormente analizadas y tratadas como fuentes de información, sino que se trataron como producciones teóricas -al mismo nivel que los textos académicos-, dándoles el mismo valor de explicación sobre los fenómenos descritos.

Después de realizar las narrativas se entretejieron los aspectos teóricos y experienciales que la atravesaban junto a los materiales académicos re- 
visados, permitiendo poner en diálogo el concepto de homonacionalismo con las experiencias de las participantes. Este diálogo ha admitido apreciar coincidencias en relación con las formas de alterización racial y diferencias en relación con la reproducción de patrones hetero y homonormativos. Las reflexiones a continuación, de carácter local y situado, permiten llevar a la reflexión y asientan la base para próximas investigaciones más amplias.

Las participantes pertenecían a diferentes colectivos con posiciones diversas respecto a la independencia de Cataluña y a la experiencia de trabajo con migración. El pseudónimo y caracterización de las participantes fue el siguiente (los nombres utilizados en este artículo son pseudónimos): (a) Ramón, de un colectivo LGT de orientación independentista, obrera y con amplia implantación en Cataluña, que trabaja temas de migración y estaría dentro de la categoría de "transformador"; (b) Eva, de un colectivo LGTI de orientación independentista y queer, fundado a finales de la década de los años 2000 y que incorpora activistas de otros colectivos, de amplia implantación en Cataluña y entre las categorías queer; (c) Laura, de un colectivo LGTB con amplia representación y experiencia en el mundo universitario catalán, sin una posición clara frente a la migración o la independencia y dentro de la categoría normalizadora y (d) Álex, de un colectivo que trabaja específicamente con otros LGTB migrantes, no posicionada en relación con la independencia, con actividad a nivel estatal e internacional y que entraría en la categoría normalizadora.

\section{Presencia del discurso homonacionalista}

Los derechos de los colectivos LGTBI han pasado a formar parte de la agencia política tanto en el Estado español como en Cataluña, tal como se refleja en las políticas públicas desarrolladas y los marcos legislativos aprobados. Sin desmerecer su gran importancia en términos de derechos sociales, son susceptibles de ser apropiados dentro de un discurso homonacionalista que sitúa a Cataluña en la parte positiva del polo colonial, donde se contrapone civilización y barbarie. Las participantes reconocen la presencia de un discurso homona- cionalista que diferencia entre autóctonos e inmigrantes en términos de discriminación homófoba, una diferenciación que se aplica principalmente a migrantes árabes:

"Yo vengo escuchando desde hace muchos años el tema de que los inmigrantes son homófobos. Pero te puedo decir que he recibido más 'insultos' por el tema LGTBI de gente autóctona que de personas, por ejemplo, islámicas, que es el prejuicio que he escuchado más" (Álex).

Vemos cómo el fragmento identifica un discurso oficialista que presenta a unas otras, "islámicas", como homófobas frente a la población autóctona. A este discurso se le opone un discurso de carácter local y experiencial que lo está negando. El discurso oficialista tendría carácter racista y excluyente a la vez que incluye al colectivo homosexual, anteriormente excluido (Lamble, 2014). Álex, además, añade que este prejuicio hacia personas árabes porque forman parte de una cultura muy diferente a la local, ya que:

"la sociedad se fija más en lo extraño, lo más visible, [...] y como la sociedad es tan ignorante, asemeja todos a islámicos, todo lo que pueda ser extraño, 'estos son árabes', o con los pakistaníes, les parecen como raros".

Para Álex, pues, la diferencia es un factor clave, y el hecho de que ciertas poblaciones se vean como más cercanas hace que haya otros tipos de prejuicios, como con la población de origen latinoamericano: "yo creo que los latinoamericanos somos concebidos como más cercanos, pero sí que hay ciertos prejuicios sobre nosotros. Sobre todo sobre los brasileños, que es que se dedican a la prostitución, o sobre las mujeres latinas, que son provocativas". Sin embargo, no identifica en su narrativa ningún prejuicio específico hacia personas de origen latinoamericano que tenga que ver con posiciones homófobas u homonacionalistas.

La oposición nosotras-ellas con base en la dimensión homófoba permite camuflar el discurso racista a la vez que construye un otro, necesariamente 
homófobo, que puede constituir un elemento de construcción identitaria para ambas comunidades. El discurso homonacionalista asume "que en temas LGBT o en feminismos [las nuevas comunidades] están un poco atrasadas, comparadas con el nivel español en general" (Laura). Para ilustrar esto, la activista explica lo siguiente:

Yo vivo en un piso, y la familia que vive enfrente son musulmanes, son inmigrantes de primera generación: un hombre, su mujer, el hermano y la hija. Y cuando fui a vivir con mi pareja, mucha gente me comentó: icómo se lo toman?, icómo os tratan?, icómo no sé qué? Y más porque nos vemos sí o sí porque normalmente están en la puerta, tienen allí la mesa, hablan, etc. Y en este sentido sí que he detectado mucho la preocupación de la gente de nuestro entorno de cómo nos tratarán. (Laura)

Al mismo tiempo, como se ilustra en el fragmento de Álex, aparece en los discursos activistas una resistencia a usar los derechos LGTB como jerarquía geopolítica. Efectivamente, a pesar de que en Cataluña hay una inclusión de los derechos LGTB en el aparato estatal, las narrativas no sostienen que se aplique una lógica colonial de "salvación desde el Primer Mundo" hacia personas de otros territorios nacionales. Si bien esto es así en los discursos activistas, no parece que suceda lo mismo en otros contextos. En este sentido, una de las participantes narra cómo en locales de ambiente se trata diferencialmente a las personas en función de su lugar de origen, aunque no especifica las procedencias donde este trato se aplica:

Se los trata un poco como inferiores, como manejables, o como si fueran más jóvenes, que tienen menos experiencia y son más ignorantes, y entonces es mucho más fácil exigirles ciertas prácticas que a otros les costaría más pedir, o negarles el uso del preservativo. (Laura)

En definitiva, algunas posiciones activistas reconocen críticamente la presencia del homonacionalismo a la vez que señalan su expansión a lo largo del tejido social, por lo que se produce una acepta- ción de la homosexualidad dentro del paradigma de modernidad occidental como eje alrededor del cual se construye el centro y la periferia.

\section{Del excepcionalismo desmovilizador a la movilización excepcionalista}

El homonacionalismo se construye a partir de incorporar la homosexualidad como elemento caracterizador de la excepcionalidad de la nación (Puar, 2007), en contraste con otros territorios geopolíticos donde los derechos LGTB no son respetados, diferenciando poblaciones en función de origen y/o etnia. Estos derechos, sin embargo, son subsidiarios respecto a la existencia y pertenencia a la nación que garantiza estos derechos y no para el conjunto de la población que habita un determinado territorio nacional. La inclusión de los derechos LGTB cubre la nación con un embalaje de modernidad y liberalidad (Currah, 2013), atractivo para la población homosexual y en contraposición a demarcaciones étnico-territoriales homófobas. El establecimiento de esta jerarquía permite desarrollar imaginarios y políticas conservadoras y, a menudo racistas hacia la población homófoba. Bajo esta perspectiva, la inclusión de los derechos de los colectivos LGTB sería secundario a la construcción nacional en lugar de constitutivo de la nación, por lo que estos derechos adquieren un carácter temporal (Puar, 2007), aspecto que se refleja en las narrativas cuando se trata la independencia de Cataluña: "con la independencia ganaríamos [la lucha LGTBI] mientras les hiciera falta tener estos votos. Es captar socios, es publicidad" (Eva). Esto tiene sentido con el poco despliegue que tiene la ley catalana contra la LGTBfobia; meses después de su aprobación todavía no se está aplicando en su totalidad (Domènech, 29 de enero de 2015).

La instrumentalización estatal de los derechos LGTB puede llevar a una actitud celebratoria ante la aparente inclusión de estos en el cuerpo de la nación, que dé lugar a una desmovilización cuando el Estado-nación asuma los objetivos políticos de dichas asociaciones (Currah, 2013). En este caso, sin embargo, precisamente se esgrime como prueba de un movimiento movilizador, el carácter excepcio- 
nal de la ley catalana. Efectivamente, los distintos colectivos han participado enérgicamente en las actividades relacionadas con la ley para garantizar los derechos de lesbianas, gais, bisexuales, transgénero e intersexuales y para erradicar la homofobia, la bifobia y la transfobia, que fue aprobada el 2 de octubre de 2014 por el parlamento de Cataluña. En las narrativas, se expresa claramente la visión de que "el pensamiento de que ciertos avances legales, como el matrimonio homosexual, estancan el movimiento es un equívoco" (Ramón), y que "en Cataluña somos unos privilegiados, pero aun así hay muchísimo trabajo por hacer" (Laura). A pesar de que ciertas leyes por los derechos LGTB puedan tener efectos necropolíticos (Lamble, 2014), lo cierto es que no deja de ser una forma de movilización.

Al mismo tiempo, los movimientos activistas señalan los efectos desmovilizadores de la incorporación de las demandas LGTBI dentro del aparato estatal (Petit, marzo de 2006), efecto que se relaciona con proceso propio, institucionalizador del movimiento LGTBI (Aljama \& Pujol, 2013). En el caso de Barcelona, la manifestación del Orgullo Gay del 28 de junio de 2011 puede servir como indicador del efecto desmovilizador de la institucionalización de los derechos LGTB, al pasar de una única manifestación de carácter político a dos manifestaciones, una de carácter político y otra de carácter comercial y lúdica, y donde esta última cuenta cada vez con más afluencia de público en detrimento de la primera. Es posible, en este sentido, que los grupos activistas políticos hayan ofrecido una versión más positiva de su situación dado el contexto de "excepcionalidad", desde el que se ha constituido la aprobación de la ley contra la homofobia.

Las narrativas, de este modo, alertan del riesgo desmovilizador de la institucionalización de las demandas de derechos para los colectivos LGTBI, a la vez que se refleja un excepcionalismo sexual, en este caso, dentro de la movilización, es decir, una movilización excepcionalista, que sería bastante diferente del excepcionalismo descrito por varias autoras. En lugar de presentar una visión que, con la situación legal y social pareciera lo suficientemente buena como para dejar de movilizarse, lo que reflejan las narrativas es que en Cataluña sigue habiendo una movilización por parte de activistas, aunque las activistas dan cuenta de que, como se describe por diferentes autoras en otros contextos, la mirada hacia ciertas comunidades respecto a los temas LGTB sigue siendo de una sensación de excepcionalidad. Esta es una diferencia sobre la que sería necesario profundizar en futuras investigaciones, teniendo en cuenta que podría ser una especificidad del contexto de Cataluña.

\section{Desafiando la homonormatividad}

El homonacionalismo, en tanto que institucionalización del discurso de liberación homosexual, participa de las formas de regulación corporal de la nación, situando y jerarquizando categorías corporales como género, clase, raza y sexualidad. En tanto que la nación se construye en un transfondo heteronormativo, con base en la jerarquización las dicotomías masculino/femenino hetero/homo, el homonacionalismo fija una nueva dicotomía en términos de tolerante/homófobo que instaura una homonormatividad que mantiene la heterosexualidad como sistema (Puar, 2007), que permea además la colonialidad del género (Lugones, 2007). Si el régimen heterosexual ha definido qué corporeidades son tolerables, deseables y valoradas en términos de, por ejemplo, raza/etnicidad, posición social, género, estructura familiar, orientación sexual o diversidad funcional, la homonormatividad construye una corporeidad compatible, a la vez que jerarquizada, respecto a la corporeidad heteronormativa. La institucionalización de la desidencia permite transformar los derechos en privilegios que pueden disfrutarse en ciertos puntos de la jerarquía poblacional, permitiendo reproducir formas tradicionales de normatividad con base en la presunta pérdida de privilegios. La inclusión de la corporeidad homonormativa en el cuerpo de la nación afianza la complicidad de grupos homosexuales en el señalamiento de corporeidades excluidas y abyectas. En este sentido, Puar (2007) señala que mientras que el otro homosexual es siempre blanco, el otro racial es siempre heterosexual, al considerar que la construcción de la alteridad heteronormativa se basa en la existencia de un otro homosexual, 
mientras que la alteridad homonormativa presume un otro homófobo. La expresión del privilegio homonormativo en Cataluña se refleja en colectivos como, por ejemplo, la Sectorial Gay y Lésbica de la Assemblea Nacional de Catalunya (ANC), que ha usado como argumento para la independencia de Cataluña el beneficio de la repercusión del turismo gay y lésbico (ANC, 2012). Este tipo de turismo, ampliamente criticado por varios sectores, refuerza el capitalismo heteronormativo (Puar, 2007). En este sentido, el activista Ramón nos muestra una postura firmemente anticapitalista al defender la práctica del cruising ${ }^{5}$ ante el ocio rosa ${ }^{6}$, cuando dice "creo que el cruising es una forma muy libre de ligar con la que estoy totalmente de acuerdo, porque es una de las pocas posibilidades no comerciales".

Posiblemente, en tanto que se trabajó con un discurso activista, las narrativas no muestran un posicionamiento en relación con una jerarquía corporeizada de lo social y, por el contrario, desarrollan una visión crítica respecto a la construcción de formas normativas y comerciales de identidades homosexuales. Una de las participantes comentaba, refiriéndose a las políticas de integración homosexual: "yo creo que hay dos tipos de lucha LGTB: la que busca integrarse, y por tanto formar parte de un sistema heteronormativo enfermizo, y la lucha que busca la equidad" (Eva). En otras narrativas, aparece un posicionamiento en contra del matrimonio homosexual, pilar de la homonormatividad: "Estábamos a favor del matrimonio como derecho, pero no como institución" (Ramón). Son discursos marcados por una crítica política donde se evita encajar dentro del cuerpo de la nación, cuestionando la dificultad de desarrollar unas formas de tener una vida LGTBI

5 Tipos de encuentros sexuales anónimos practicados en lugares públicos, habitualmente en parques, playas y bosques (entre otros), sobre todo cuando estos encuentros son entre hombres.

6 El ocio rosa o el capitalismo rosa hace referencia al conjunto de negocios que se destinan solamente a público homosexual (sobre todo homonormativo), dirigidos a un grupo de población de clase media y alta que antes quedaba menos instrumentalizada por el capitalismo al no haber negocios específicamente homosexuales. Son ejemplo de ocio y capitalismo rosa las discotecas, tiendas de ropa y complejos turísticos específicamente destinados a gais y lesbianas. al margen de las formas homonormativas de consumo e institucionalidad lésbico y gay. También se explicita esta dificultad cuando se habla de los discursos y acciones desarrolladas en contra del cruising: "en una sociedad con una 'democracia' como la nuestra nos encontramos con muchos intereses [comerciales...] en contra del cruising" (Ramón). También se enfatiza la importancia del diálogo intercultural como elemento fundamental en algunos colectivos que trabajan con migración LGTBI: "Quizás nuestro discurso filosófico parte de la interculturalidad, que para nosotros no es sólo el tema inmigración, sino que es la interconexión entre diferentes" (Álex). Un hablar desde la interculturalidad y la interconexión entre diferentes que cuestiona la construcción de una alteridad racial homófoba.

El discurso activista, quizá por su naturaleza crítica con las formas de poder institucional, cuestiona el establecimiento de una homonormatividad que fundamente el discurso homonacionalista. Es posible, en este sentido, que debiera distinguirse entre la excepcionalidad sexual desde posiciones que reproducen formas estatales que enaltecen la propia nación frente a otros territorios geopolíticos y la excepcionalidad sexual desde un activismo político dirigido a la transformación de las prácticas de discriminación hacia al colectivo LGTBI. Si bien es posible que haya entidades o colectivos que refuercen la homonormatividad, también identificamos prácticas y discursos de resistencia a las normas homogeneizadoras de corporeidades y formas de hacer heteronormativas.

\section{A modo de conclusión: homonacionalismo catalán frente a excepcionalidad sexual activista}

En este trabajo hemos explorado el potencial crítico del concepto de homonacionalismo en relación con el contexto catalán. En esta primera aproximación, hemos mostrado la necesidad de utilizar esta herramienta de análisis para ver cómo el repliegue de ciertas posiciones de sujeto LGTB se utiliza instrumentalmente en Cataluña y algunos de los efectos que esto supone. 
En la investigación realizada hemos podido ver cómo algunas activistas sostienen que en Cataluña está presente una posición excepcionalista, que supone una construcción de alteridad alrededor de la aceptación o no de la homosexualidad. En este sentido, la excepcionalidad sexual en Cataluña toma lugar además de la movilización, y no en contraposición a ella, pudiendo hablar de una movilización excepcionalista. La movilización en el territorio catalán sigue activa, ejemplo de ello es la reciente creación de una ley contra la LGTBfobia, aunque siguen reproduciéndose en el territorio estereotipos y visiones coloniales frente a determinadas subjetividades migrantes.

Las activistas participantes expresan también una clara posición de resistencia a la homonormalización, porque sostienen discursos anticapitalistas, antirracistas o antinstitucionales. Esta posición, que entra en conflicto con otros desarrollos del concepto de homonacionalismo, es una clara resistencia al mismo que sería interesante seguir estudiando en futuros trabajos. Si bien en las narrativas se muestra cómo ciertas posiciones toman parte del capitalismo rosa y de la asimilación a un sistema heteronormativo, hay claramente movilización activa frente a estas posiciones.

Por otro lado, las narrativas muestran cómo algunas formas de configuración de la nación catalana toman lógicas homonacionalistas. La percepción de la inclusión temporal de los derechos LGTB, así como el hecho que la ley contra la LGTBfobia todavía no esté del todo implementada, son una muestra de que bajo la aparente voluntad del Gobierno para incluir discursos y luchas en la política principal no existe una voluntad política para la inclusión real de subjetividades disidentes.

En esta primera aproximación al estudio del homonacionalismo en Cataluña, se puede apreciar la necesidad de seguir indagando en las articulaciones entre discursos y prácticas homonacionalistas en el contexto catalán. Aunque las narrativas desarrollan un planteamiento crítico hacia la inclusión del discurso de liberación homosexual en la política y la sociedad catalanas, se trata de participantes con tendencia política y sería posible esperar posiciones más cercanas a la institucionalización homonacionalista en otros grupos sociales.
Finalmente, es necesario destacar que la crítica a la coaptación institucional del discurso LGTBI se debería desarrollar con el máximo cuidado y trato ético posible. La crítica homonacionalista es susceptible de desvirtuar el trabajo hecho por los colectivos desde hace décadas. Sin embargo, no debemos detener el potencial crítico del concepto de homonacionalismo, para así evitar que los derechos y libertades de ciertos grupos poblacionales se construyan a expensas de los derechos y marginalización de otros grupos.

\section{Referencias}

Adorno, T. W. (1990). Dialéctica negativa. Madrid: Taurus.

Adorno, T. W. (1991). Actualidad de la filosofía. Barcelona: Paidós.

Aljama, P., \& Pujol, J. (2013). Reflexiones sobre la institucionalización del movimiento LGBT desde el contexto catalán y español. Interface, 5(2), 159-177.

Ammaturo, F. R. (2014). The right to a privilege? Homonormativity and the recognition of same-sex couples in Europe. Social Eु Legal Studies, 23(2), 175 194. http://dx.doi.org/10.1177/0964663914521651

Assemblea Nacional de Catalunya. Sectorial de Gais i Lesbianes per la Independència. (2012). Document Constitutiu. Barcelona: Autor. Recuperado de https://assemblea.cat/sites/default/files/dos_sectorials/DocumentConstitutiuGLxIndep\%20-pdf

Andrade, B. (2014). Homonacionalismo e cidadania LGBT em tempos de neoliberalismo: dilemas e impasses às lutas por direitos sexuais no Brasil. Em Pauta, 34(12), 155-178.

Anthias, F. (1999). Beyond unities of identity in high modernity. Identities, 6(1), 121-144. http://dx.doi.or g/10.1080/1070289X.1999.9962638

Balasch, M., \& Montenegro, M. (2003). Una propuesta metodológica desde la epistemología de los conocimientos situados: las producciones narrativas. Encuentros en Psicología Social, 1(3), 44-48.

Bourdieu, P. (1986). The forms of capital. En J. Richardson (Ed.), Handbook of theory and research for the sociology of education (pp. 241-258). Nueva York: Greenwood Press. 
Bourdieu, P. (1996). The rules of art: Genesis and structure of the literary field. Stanford: Stanford University Press.

Bourdieu, P. (2002). Razones prácticas. Sobre la teoría de la acción. Barcelona: Anagrama.

Castells, M. (1999). La era de la información: economía, sociedad y cultura. Madrid: Siglo XXI.

Colpani, G., \& Habed, A. J. (2014). What is European about homonationalism? Thinking through the Italian Case. En K. Leurs \& D. Olivieri (Eds.), Everyday feminist research praxis: Doing gender in the Netherlands (pp. 23-39). Newcastle: Cambridge Scholars Publishing.

Crenshaw, K. (1991). Mapping the margins: Intersectionality, identity politics, and violence against women of color. Stanford Law Review, 43(6), 1241 1299. http://dx.doi.org/10.2307/1229039

Curiel, O. (2007). Crítica poscolonial desde las prácticas políticas del feminismo antirracista. Nómadas, 26, 92-101.

Currah, P. (2013). Homonationalism, State rationalities, and sex contradictions. Theory $\mathscr{E}$ Event, 16(1). Recuperado de http://muse.jhu.edu/journals/ theory_and_event/ v016/16.1.currah.html

Domènech, F. (29 de enero de 2015). 100 dies després de l'aprovació de la llei contra la LGTBfòbia la Generalitat encara no n'ha fet el desplegament. $\mathrm{La}$ Directa. Recuperado de www.directa.cat

Domingo, A. (2013). Catalonia, land of immigration. En L. Castro (Ed.), What's up with Catalonia? Ashfield, MA.: Catalonia Press.

Durand Ponte, V. M., \& Cuéllar Vázquez, M. A. (1989). Clases y sujetos sociales : un enfoque crítico-comparativo. México: UNAM.

El-Tayeb, F. (2012). 'Gays who cannot properly be gay': Queer Muslims in the neoliberal European city. European Journal of Women's Studies, 19(1), 79-95. http://dx.doi.org/10.1177/1350506811426388

Ferreira, G. B. (2014). Ativistas, artistas e queers no sul global: ainda nas margens do mujeres al borde. Revista Gênero na Amazônia, 6, 91-115.

Gil Calvo, E. (2006). La ideología española. Oviedo: Ediciones Nobel.

Gramsci, A. (1975). Cartas desde la cárcel (Vol. 82). Madrid: Cuadernos para el Diálogo.
Gunn, S. (2006). From hegemony to governmentality: Changing conceptions of power in social history. Journal of Social History, 39(3), 705-720.

Haraway, D. J. (1991). Ciencia, cyborgs y mujeres. La reinvención de la naturaleza. Madrid: Cátedra.

Haritaworn, J. (2013). Beyond 'hate': Queer metonymies of crime, pathology and anti/violence. Jindal Global Law Review, 4(2), 44-78.

Laclau, E., \& Mouffe, C. (1985). Hegemonía y estrategia socialista. Madrid: Siglo XXI.

Lamble, S. (2014). Queer investments in punishment: Sexual citizenship, social movements and the expanding carceral state. En J. Haritaworn, A. Kuntsman \& S. Posocco (Eds.), Queer necropolitics (pp. 151-171). Oxford, UK: Routledge.

Lugones, M. (2007). Heterosexualism and the colonial/ modern gender system. Hypatia, 22(1), 186-209.

Martínez-Guzmán, A., \& Montenegro, M. (2010). Narrativas en torno al Trastorno de Identidad Sexual. De la multiplicidad transgénero a la producción de trans-conocimientos. Prisma Social, 4, 1-44.

Petit, J. (marzo, 2006). Reflexiones de presente y futuro para el movimiento LGBT. Trabajo presentado en la Jornada contra la homofòbia, Asamblea General de la CGL, Barcelona, España. Recuperado de http://www.amicsgais.org/documents/ Reflexiones de futuro-JP.pdf

Puar, J. K. (2006). Mapping US homonormativities. Gender, Place Ë Culture, 13(1), 67-88. http://dx.doi. org/10.1080/09663690500531014

Puar, J. (2007). Terrorist assemblages: Homonationalism in queer times. Durham: Duke University Press Books.

Puar, J. (2013). Rethinking homonationalism. International Journal of Middle East Studies, 45(2), 336-339. http://dx.doi.org/10.1017/S002074381300007X

Pujol, J., Montenegro, M., \& Balasch, M. (2003). Los límites de la metáfora lingüística. Implicaciones de una perspectiva corporeizada para la práctica investigadora e interventora. Política y Sociedad, $40(1), 57-70$

Roig Tapia, A. (2009). Memoria de la violencia. Transición, consolidación y crispación democrática en España (1975-2008). En S. Baby, O. Compagnon \& E. González Calleja (Coords.), Violencia y transiciones políticas a finales del siglo XX: Europa del 
Sur-América Latina (pp. 41-58). Madrid: Casa de Velázquez.

Seguer, L. (2014). De la normatividad queer en la construcción de la nación a la resistencia política queer: un debate en la relación Israel-Palestina. Universitas Humanística, 78(78), 261-280.

Townson, N. (Dir.). (2011). iEs España diferente?: una mirada comparativa. Barcelona: Taurus.
Valentine, G. (2007). Theorizing and researching intersectionality: A Challenge for feminist geography. The Professional Geographer, 59(1), 10-21. doi:10.1111/j.1467-9272.2007.00587.x

Wacquant, L. (2006). Key contemporary thinkers. Londres/Nueva York: Macmillan. 
